\title{
The GPR early-time method to measure water content of clay soils
}

\author{
Remke L. Van Dam ${ }^{1,2,3}$, Jonathan Algeo ${ }^{4}$, and Lee Slater ${ }^{4}$ \\ ${ }_{1}^{1}$ Queensland University of Technology, Institute for Future Environments, Brisbane, QLD, Australia. \\ ${ }^{2}$ Michigan State University, Department of Earth and Environmental Sciences, East Lansing, MI, USA. \\ ${ }^{3}$ Now at: CEFET-MG, Programa de Pós-Graduação em Engenharia Civil, Belo Horizonte, MG, Brazil. \\ ${ }^{4}$ Rutgers University, Department of Earth and Environmental Sciences, Newark, NJ, USA.
}

Copyright 2016, SBGf - Sociedade Brasileira de Geofísica

Este texto foi preparado para a apresentação no VII Simpósio Brasileiro de Geofísica, Ouro Preto, 25 a 27 de outubro de 2016. Seu conteúdo foi revisado pelo Comitê Técnico do VII SimBGf, mas não necessariamente representa a opinião da SBGf ou de seus associados. É proibida a reprodução total ou parcial deste material para propósitos comerciais sem prévia autorização da SBGt.

\begin{abstract}
Ground-penetrating radar (GPR) early-time amplitude analysis has recently been proposed as an alternative way for measuring soil water content (SWC) in the shallow subsurface. The method uses amplitude attributes of overlapping GPR air- and ground-waves of bi-static antennas, which have been shown to vary with soil dielectric properties. We tested the feasibility of this approach to characterize soil water content at clay-rich sites. An irrigation experiment was conducted in a natural grassland at the Samford Ecological Research Facility in Queensland, Australia. We collected daily GPR and timedomain reflectometry (TDR) datasets over a five-day period and collected samples for gravimetric moisture analysis. The GPR data display a clear response of the early-time signal amplitude to spatial and temporal changes in SWC. Our results show that the early-time methodology can successfully characterize soil water content at clay-rich sites, where (due to high signal attenuation) traditional GPR methods typically fail.
\end{abstract}

\section{Introduction}

Root-zone soil water content (SWC) is a key driver of climate, energy and carbon cycles, and is critical to ecosystem dynamics, agricultural water management and biological and chemical processes in the vadose zone. Understanding the spatial distribution and temporal dynamics of soil moisture at a range of scales is thus of paramount importance (e.g., Robinson et al., 2008). Traditional methods for assessment of soil moisture can provide high quality time series but these measurements are often invasive and point-based, and cannot readily be up-scaled (e.g., Dorigo et al., 2011). In contrast, remote sensing techniques provide regional-scale estimates of water content, but these often have limited spatial and temporal resolution (e.g., Loew et al., 2013). Therefore, to measure SWC at the field scale there is a need for alternative tools.

Over the past two decades, ground-penetrating radar (GPR) has become a much-used tool for research of SWC variation at the field scale. Typical approaches include collection of GPR reflection, common-mid-point (CMP) and ground-wave data (Huisman et al., 2003). The practical use of GPR is somewhat limited, however, due to the required setup with large antenna spacings. Moreover, the performance of the GPR method is negatively impacted by conductive soils, such as those that contain saline pore fluids or have a high clay content, both of which attenuate the signal.

The recently proposed GPR early-time methodology provides an alternate way to assess SWC at the field scale (Pettinelli et al., 2007). These researchers demonstrated that there is a correlation between some characteristics of the overlapping direct air- and ground-waves (the earlytime signal) and the dielectric properties of the nearsurface soil. From widely-used pedotransfer functions it is known that these dielectric property variations, measured in the early-time signal, can then be related to SWC (e.g., Van Dam, 2014).

The early-time method has the benefit that transmitter and receiver antennas do not need to be separated, as is required for CMP and ground-wave measurements. The method can thus be used with commercial bistatic antenna systems with small antenna offsets. This also means that the early-time method may provide a solution to conductive field sites, where excessive signal attenuation typically renders GPR ineffective. One disadvantage of the earlytime method is the limited depth of investigation.

In this work, we present a field experiment to characterize the spatial and temporal variability in the early-time signal attributes using time-lapse GPR measurements before and after an irrigation event. The results are compared with two independently obtained values of SWC. The experiment was conducted in a natural grassland with a clay-rich soil in Queensland, Australia.

\section{The Early-time GPR Method}

The GPR technique is based on the transmission and propagation of electromagnetic (EM) energy in the subsurface. Frequency dependent material properties that affect the behaviour of this energy include the dielectric permittivity, electrical conductivity, and magnetic permeability. Together, these properties define the EM impedance of the medium.

A GPR measurement typically involves a transmitting antenna that emits a radar pulse, and a receiving antenna that is used to record characteristics of the arriving energy. The pulse travels at a velocity that largely depends on the relative dielectric permittivity $\left(\varepsilon_{r}\right)$ of the medium it traverses. Because $\varepsilon_{r}$ of fresh water is around 80 , while for air and for mineral soil grains it is 1 and around 4 , respectively, small changes in pore water content can lead to significant variations in bulk dielectric properties. 
In GPR early-time analysis, the earliest part of the signal in a common-offset survey (where ground- and air waves typically overlap) is analyzed. First, a Hilbert Transform is performed on the signal. Next, the envelope of the trace, also known as the instantaneous amplitude, is calculated (Figure 1), by taking the absolute value of the transformed GPR signal (e.g., Ferrara et al., 2013).

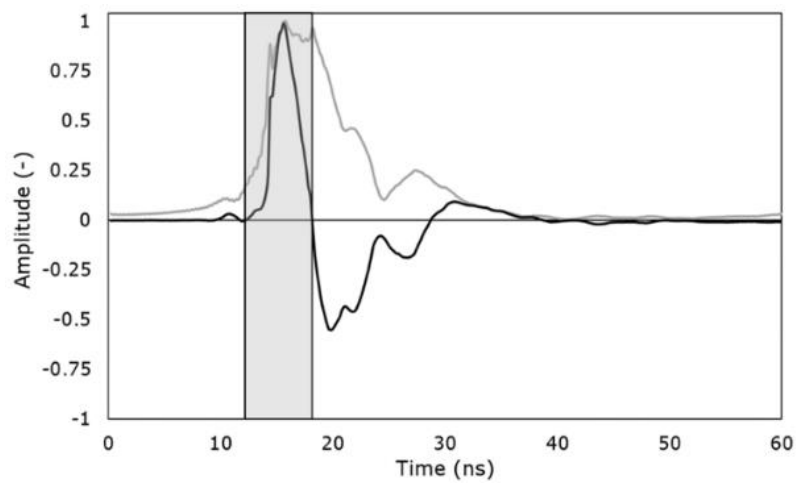

Figure 1 - Representative GPR trace and corresponding envelope (gray line). The shaded area highlights the first positive half cycle.

A custom function is used to extract the first positive half cycle of each measurement (see Figure 1). This portion of the early-time signal has the greatest signal to noise ratio and has a good correlation with the soil dielectric properties (Di Matteo et al., 2013). The integral amplitude of the first positive half cycle is then divided by the unit length of the integral, which gives the average envelope amplitude (AEA). For consistency with the work of Pettinelli et al. (2007) and Di Matteo et al. (2013), this value is then inverted to give $A E A^{-1}$.

The $\mathrm{AEA}^{-1}$ values are arbitrary "amplitude units", which means that they cannot be directly converted into dielectric properties or SWC. An alternative methodology that may allow estimation of an absolute value of SWC from the early-time signal was presented by Hislop (2015), but this method has not yet been tested for clays.

It is also worth noting that the early-time signal is affected by both bulk conductivity and dielectric permittivity but that their effects cannot yet be separated. However, it has been suggested that for typical site conditions the permittivity affects the early-time amplitude to a greater extent than conductivity. Also, changes in conductivity do not affect the strength of correlation between early-time amplitude and changes in permittivity (Di Matteo et al., 2013).

\section{Experimental Setup}

The Samford Ecological Research Facility (SERF) is a 51hectare property in Queensland, Australia (Figure 2) with a large portion maintained as a typical Australian subtropical grassland. The soil at the site contains up to $40 \%$ clay in some horizons. This study focused on an area in the northwest corner of SERF's grassland, where it is relatively flat and water for irrigation was available.
The experiment was conducted over a 20 by $14 \mathrm{~m}$ grid (Figure 2). For the GPR surveys, 15 transects with $1 \mathrm{~m}$ spacing were marked with guide lines. 101 locations were designated for repeat time-domain reflectometry (TDR) measurements. Following the experiment, at 12 of these locations duplicate soil samples were taken at $5-10 \mathrm{~cm}$ and $15-20 \mathrm{~cm}$ depths (4 samples total) for measurement of the gravimetric water content (GWC).

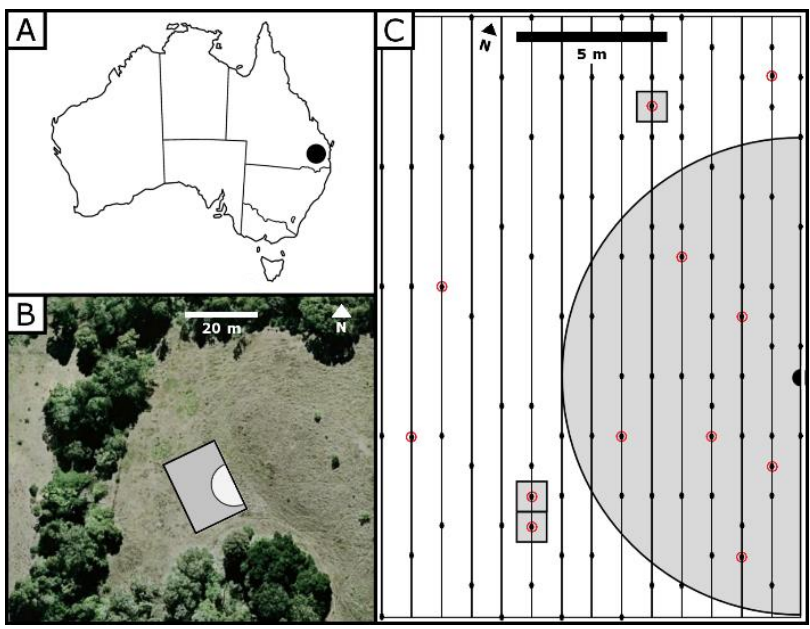

Figure 2-a) Location of SERF in Australia. b) Position of the grid at the field site. c) Setup of the $14 \times 20 \mathrm{~m}$ grid. Vertical lines represent GPR transects, black circles are $T D R$ locations, and red circles represent soil sampling locations. The gray semi-circle is the sprinkler-irrigated area, and the squares represent box infiltrometers.

Background GPR and TDR datasets were collected on Day 1 of the experiment. After this, the grid was irrigated overnight using a garden sprinkler set along the edge of the grid, wetting the grid to a distance of around $8 \mathrm{~m}$ (Figure 2 ). In addition, three $1 \mathrm{~m}^{2}$ box infiltrometers were used to provide an alternate type of wetting. During four subsequent days (Days 2-5), time-lapse GPR and TDR data sets were collected at the site.

GPR data were collected using a PulseEKKO 1000 system with $200 \mathrm{MHz}$ antennas, mounted in a SmartCart at $0.5 \mathrm{~m}$ separation. The cart was equipped with an odometer wheel to accurately position measurements along the transects. TDR measurements were made using a HydroSense system, which outputs results as volumetric water content (VWC) values. The TDR probe had two $12 \mathrm{~cm}$ rods that were vertically inserted into the soil.

\section{Results}

Figure 3 presents the results of the early-time GPR analysis. The plots show $\mathrm{AEA}^{-1}$ of the first positive half cycle derived from averaging blocks of 7 traces. The Day 1 background dataset, collected before irrigation, has an average $\mathrm{AEA}^{-1}$ value of $5.91 \cdot 10^{-5}$ (Figure $3 \mathrm{a}$ ). The few spots with higher $A E A^{-1}$ values are from rainwater that bypassed the tarp covering the grid prior to the experiment. 


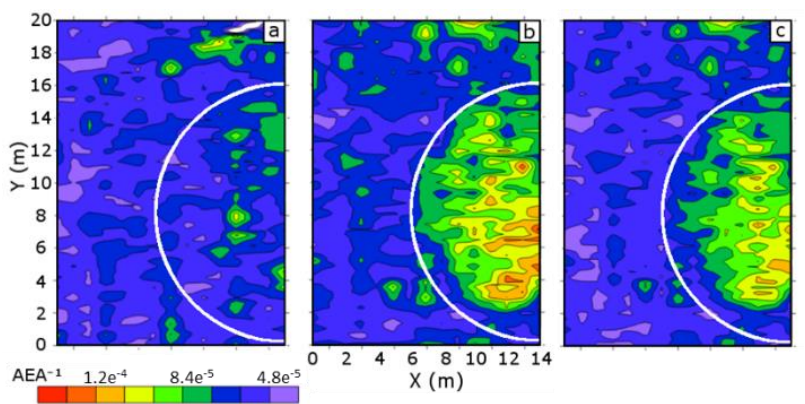

Figure 3 - Maps of GPR AEA $A^{-1}$ collected on Day 1 (before irrigation), Day 2, and Day 5. The grids were obtained via kriging, using a linear variogram model (slope $=1$ ).

The values on Day 2, the first dataset collected after irrigation, are mostly unchanged in the non-irrigated area (Figure 3b). However, in the area of sprinkler irrigation, the $\mathrm{AEA}^{-1}$ values increase to a range of $0.72-1.36 \cdot 10^{-4}$ (the field site average is $\left.7.10 \cdot 10^{-5}\right)$. By Day 5 , the $A E A^{-1}$ values in the irrigated area have notably decreased (the field site average is $6.52 \cdot 10^{-5}$; Figure $3 c$ ), reflecting the gradual drying process of the soil. The locations of the box infiltrometers do not register a strong response in $A E A^{-1}$ values, which we believe is partly due to the fact that traces were averaged in groups of 7 before processing.

The TDR data collected during the experiment are in general agreement with the GPR early-time data, where an increased VWC corresponds with an increase in $A E A^{-1}$. The data in Figure 4a show this relationship for Day 5 of the experiment.

The GPR early-time data also correlate well with the GWC values obtained from the soil samples collected on Day 5 (Figure 4b). The correlation is strongest for the samples collected over the $5-10 \mathrm{~cm}$ depth range, which suggests a depth-of-investigation for the early-time method (for this soil and using $200 \mathrm{MHz}$ antennas) of around $10 \mathrm{~cm}$.

\section{Conclusions}

This irrigation experiment demonstrates the viability of the early-time GPR method for estimating SWC in electrically conductive soils, where other GPR methods typically fail due to high signal attenuation. The data show a strong correlation between the GPR early-time values and two independently obtained measures of SWC. These correlations suggest that GPR early-time $A A^{-1}$ values may be converted to SWC via simple (linear) regression.

Our ongoing studies include the depth of investigation of the early-time method and the individual effects of electrical conductivity and permittivity on the signal.

\section{Acknowledgements}

The research was supported by a U.S. National Science Foundation and Australian Academy of Science grant, awarded to Jon Algeo (East Asia and Pacific Summer Institutes for U.S. Graduate Students), and by the Institute for Future Environments at QUT. We thank Professor Peter Grace for his support; Marcus Yates and Nicholas Josephs provided field assistance.
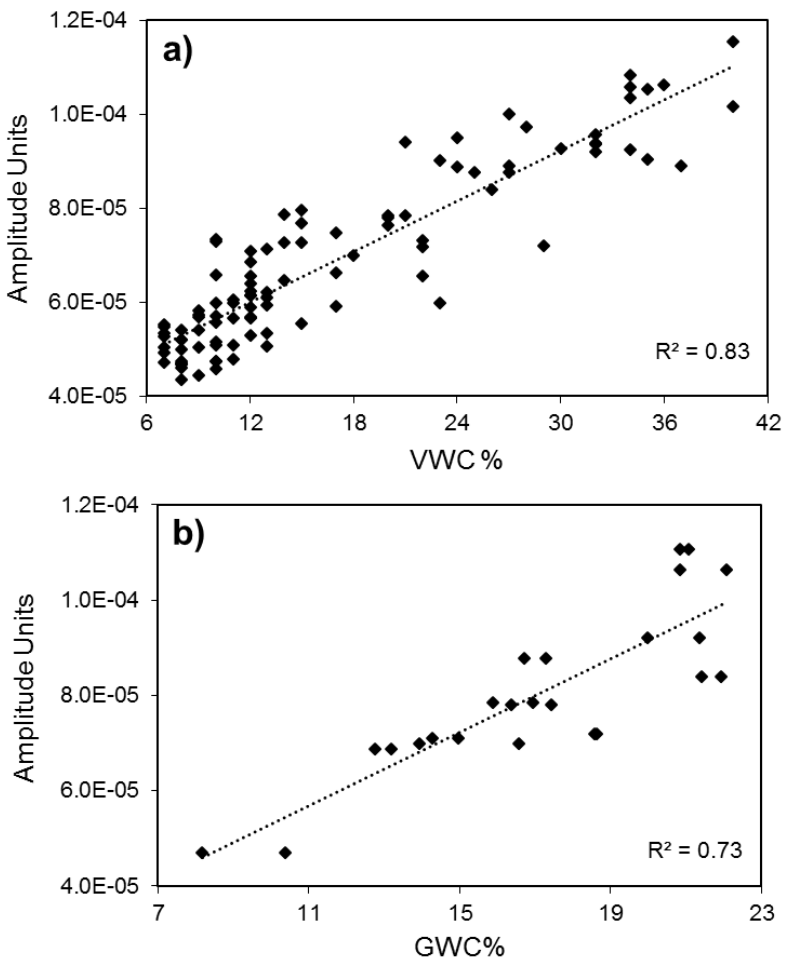

Figure 4 - GPR $A E A^{-1}$ on Day 5 versus a) volumetric water content from 101 TDR measurements, and b) gravimetric water content from 24 soil samples collected at 12 locations.

\section{References}

Di Matteo, A., Pettinelli, E., Slob, E., 2013. Early-time GPR signal attributes to estimate soil dielectric permittivity: $A$ theoretical study. IEEE Transactions in Geoscience and Remote Sensing, 51.3: 1643-1654.

Dorigo, W. A., Wagner, W., Hohensinn, R., Hahn, S., Paulik, C., Xaver, A., Gruber, A., Drusch, M., Mecklenburg, S., van Oevelen, P., Robock, A., and Jackson, T., 2011. The International Soil Moisture Network: A data hosting facility for global in situ soil moisture measurements. Hydrology and Earth System Sciences, 15: 1675-1698.

Ferrara, C., Barone, P. M., Steelman, C. M., Pettinelli, E., and Endres, A. I., 2013. Monitoring shallow soil water content under natural field conditions using the early-time GPR signal technique. Vadose Zone Journal, 12.

Hislop, G., 2015. Permittivity estimation using coupling of commercial ground penetrating radars." IEEE Transactions in Geoscience and Remote Sensing 53: 4157-4164.

Huisman, J. A., Hubbard, S. S., Redman, J. D., and Annan, A. P., 2003. Measuring soil water content with ground penetrating radar. Vadose Zone Journal, 2: 476-491.

Loew, A., Stacke, T., Dorigo, W., de Jeu, R., and Hagemann, S., 2013. Potential and limitations of multidecadal satellite soil moisture observations for 
selected climate model evaluation studies. Hydrology and Earth System Sciences, 17, 3523-3542.

Pettinelli, E., Vannaroni, G., Di Pasquo, B., Mattei, E., Di Matteo, A., De Santis, A., Annan, A. P., 2007. Correlation between near-surface electromagnetic soil parameters and early-time GPR signals: An experimental study. Geophysics, 72: A25-A28.

Robinson, D. A., Campbell, C. S., Hopmans, J. W., Hornbuckle, B. K., Jones, S. B., Knight, R., Ogden, F., Selker, J., and Wendroth, O., 2008. Soil moisture measurement for ecological and hydrological watershedscale observatories: A review. Vadose Zone Journal, 7: 358-389.

Van Dam, R. L., 2014. Calibration functions for estimating soil moisture from GPR dielectric constant measurements. Communications in Soil Science and Plant Analysis, 45: 392-413. 